\title{
Stability of rapid maxillary expansion and facemask therapy: A long-term controlled study
}

\author{
Caterina Masucci, ${ }^{a}$ Lorenzo Franchi, ${ }^{b}$ Efisio Defraia, ${ }^{c}$ Manuela Mucedero, ${ }^{d}$ Paola Cozza, ${ }^{\mathrm{e}}$ and Tiziano Baccetti ${ }^{\mathrm{b}}$ \\ Florence and Rome, Italy
}

\begin{abstract}
Introduction: The aim of this prospective controlled study was to evaluate the long-term effects of rapid maxillary expansion and facemask therapy in Class III subjects. Methods: Twenty-two subjects ( 9 boys, 13 girls; mean age, 9.2 years \pm 1.6 ) with Class III disharmony were treated consecutively with rapid maxillary expansion and facemask therapy followed by fixed appliances. The patients were reevaluated at the end of the 2-phase treatment (mean age, 14.5 years \pm 1.9 ) and then recalled about 8.5 years after the end of rapid maxillary expansion and facemask treatment (mean age, 18.7 years \pm 2.1 ). Two groups of controls with untreated Class III malocclusion were used for statistical comparisons of the short-term and long-term intervals. Statistical comparisons were performed with the Mann-Whitney $U$ test. Results: In the long term, no significant differences in maxillary changes were recorded, whereas the treatment group showed significantly smaller increases in mandibular protrusion. The sagittal maxillomandibular skeletal variables maintained significant improvements in the treatment group vs the control groups. Conclusions: In the long term, rapid maxillary expansion and facemask therapy led to successful outcomes in about $73 \%$ of the Class III patients. Favorable skeletal changes were mainly due to significant improvements in the sagittal position of the mandible. (Am $\mathrm{J}$ Orthod Dentofacial Orthop 2011;140:493-500)
\end{abstract}

$\mathrm{R}$ apid maxillary expansion and facial mask (RME/ FM) therapy is the most common orthopedic treatment protocol for Class 111 malocclusion. ${ }^{1,2}$ The literature includes many articles on the short-term results of RME/FM therapy in growing subjects with Class 111 disharmonies, as described in a recent systematic review. ${ }^{3}$ Several studies have also evaluated the outcomes of the orthopedic treatment protocol at postpubertal observations after fixed appliance therapy, either with $^{4}$ or without ${ }^{5,6}$ untreated Class 111 controls. Both short-term and postpubertal observations indicated

${ }^{a}$ Research associate, Department of Orthodontics, University of Florence, Florence, ltaly.

${ }^{\mathrm{b}}$ Assistant professor, Department of Orthodontics, University of Florence, Florence, Italy; Thomas M. Graber Visiting Scholar, Department of Orthodontics and Pediatric Dentistry, School of Dentistry, University of Michigan, Ann Arbor. ${ }^{\mathrm{c}}$ Associate professor and department chair, Department of Orthodontics, University of Florence, Florence, Italy.

${ }^{\mathrm{d}}$ Research fellow, Department of Orthodontics, University of Rome Tor Vergata, Rome, ltaly.

'eProfessor and department chair, Department of Orthodontics, University of Rome Tor Vergata, Rome, Italy.

The authors report no commercial, proprietary, or financial interest in the products or companies described in this article.

Reprint requests to: Tiziano Baccetti, Department of Orthodontics, University of Florence, Via del Ponte di Mezzo, 46-48, Florence, Italy 50127; e-mail, tbaccetti@unifi.it.

Submitted, April 2010; revised and accepted, September 2010. 0889-5406/\$36.00

Copyright (C) 2011 by the American Association of Orthodontists.

doi:10.1016/j.ajodo.2010.09.031 a fair-to-good effectiveness of RME/FM therapy, with about $70 \%$ to $80 \%$ of the patients showing favorable results after puberty. The dentoskeletal changes induced by therapy consist of a combined effect of the protocol on both maxillary and mandibular components. Optimal timing for the orthopedic approach to Class 111 malocclusion is related to early treatment, at either a prepubertal or a pubertal phase of development. ${ }^{7}$

No data are available in the literature with regard to the outcomes of RME/FM therapy revaluated at the end of active craniofacial growth-ie, after the circumpubertal developmental period. This information is vital for the appraisal of orthopedic treatment results in patients with Class 111 malocclusion for at least 2 main reasons. First, a significant tendency for the reestablishment of the Class 111 growth pattern has been widely demonstrated after active protraction therapy, with special emphasis during the pubertal growth spurt. ${ }^{4,8-10}$ Second, pubertal growth tends to last longer in Class 111 subjects compared with Class 1 subjects. ${ }^{11}$ On the other end, long-term observations at the end of active craniofacial growth are available for different orthopedic and orthodontic approaches: chincup therapy, with favorable short-term changes often not maintained at the end of growth, ${ }^{12}$ and mandibular cervical headgear, with greater long-term stability of favorable mandibular modifications. ${ }^{13}$

The aim of this study was to analyze the long-term outcomes of RME/FM therapy in Class 111 subjects. The 
study included Class 111 subjects treated consecutively with the orthopedic protocol in a prospective design and subjects with untreated Class 111 malocclusions as controls.

\section{MATERIAL AND METHODS}

A sample of 30 subjects with Class 111 dentoskeletal disharmony were treated consecutively with RME/FM therapy followed by comprehensive preadjusted edgewise therapy at the Departments of Orthodontics of the University of Florence and the University of Rome Tor Vergata. All patients had the following dentoskeletal features before therapy (T1) when the pretreatment lateral cephalogram was taken: European ancestry (white), anterior crossbite or edge-to-edge incisor relationship, Class 111 molar relationship, Wits appraisal of $-2.0 \mathrm{~mm}$ or less, no discrepancy between centric occlusion and centric relation (indicating pseudo-Class 111 malocclusion), and prepubertal skeletal maturation (CS 1-CS 3). ${ }^{14}$

The patients were reevaluated with a lateral cephalogram at the end of the 2-phase treatment (T2) and then recalled at an average of 4 years after T2 (T3, about 8.5 years from the end of RME/FM treatment). Eight of the 30 initial subjects could not be located at T3 or did not agree to be reevaluated radiographically in the long term. Therefore, a lateral cephalogram at T3 was taken in the 22 subjects ( 9 male, 13 female) who represented the final sample of the study. The T3 cephalograms were taken at least 2 years after the attainment of stage 6 in cervical vertebral maturation (completion of circumpubertal active craniofacial growth) for all treated subjects.

Two samples of controls with untreated Class 111 malocclusions were used for statistical comparisons of the short-term (T1-T2) and long-term (T1-T3) observation intervals. All subjects in the control groups had been followed longitudinally at the Department of Orthodontics of the University of Florence, and they matched the treated group according to ancestry, dentoskeletal Class 111 characteristics, age, skeletal maturation at all observation periods, duration of observation intervals, and sex distribution.

The 3 components of the RME/FM therapy used in this study were a maxillary expansion appliance, a facemask, and heavy elastics. ${ }^{1,15}$ Treatment began with the placement of a bonded or banded maxillary expander to which were attached vestibular hooks extending in a superior and anterior direction. The patients were instructed to activate the expander once or twice a day until the desired transverse width was achieved.

The patients were given facemasks with pads fitted to the chin and forehead for support either during or immediately after expansion. Elastics were attached from the soldered hooks on the expander to the support bar of the facemask in a downward and forward vector, producing orthopedic force levels up to 400 to $500 \mathrm{~g}$ per side. The patients were instructed to wear the facemask for a minimum of 14 hours per day. All patients were treated at least to a positive dental overjet before discontinuing treatment; most patients were overcorrected toward a Class 11 occlusal relationship. The average duration of the RME/FM treatment was 1.1 years \pm 5 months.

As occurs in studies involving any removable device, compliance with the instructions of the orthodontist and staff varied among patients. Therefore, compliance was appraised with a 3-point Likert scale (poor, moderate, good). ${ }^{16}$ Nineteen of the 22 subjects (86.3\%) underwent a second phase of preadjusted edgewise therapy after an interim period, during which a removable mandibular retractor typically was worn or, in a few instances, immediately after the rapid maxillary expansion and protraction treatment. ${ }^{17}$ On average, fixed appliance therapy lasted 18 months. During the T2-T3 period, the patients wore a standard Hawley retainer at night for about 2 years, and thereafter they wore no retention appliance.

A customized digitization regimen and cephalometric analysis provided by Viewbox (version 3.0, dHAL Software, Kifissia, Greece) was used for all cephalograms examined in this study. The customized cephalometric analysis, containing measurements from the analyses of Jacobson, ${ }^{18}$ McNamara, ${ }^{19}$ and Steiner, ${ }^{20}$ generated 19 variables, 9 angular and 10 linear, for each tracing. Magnification was standardized to an $8 \%$ enlargement for all radiographs in both treated and control samples.

\section{Statistical analysis}

Descriptive statistics were calculated for age at $\mathrm{T} 1$, $\mathrm{T} 2$, and $\mathrm{T} 3$, and for the $\mathrm{T} 1-\mathrm{T} 2$ and $\mathrm{T} 1-\mathrm{T} 3$ age intervals in all groups. Statistical comparisons on these data were performed with the Mann-Whitney $\mathrm{U}$ test. Chisquare tests were used to assess differences in sex distributions between groups. The comparison between the treated group and the controls on the dentoskeletal features at T1 (starting forms) was performed with KruskalWallis and Tukey post-hoc tests.

The following statistical comparisons were carried out with the Mann-Whitney U test: (1) treatment effects (T1-T2: T1-T2 changes in the treatment group vs T1$\mathrm{T} 2$ changes in the T1-T2 control group; and (2) overall long-term effects (T1-T3: T1-T3 changes in the treatment group vs $\mathrm{T} 1-\mathrm{T} 3$ changes in the $\mathrm{T} 1-\mathrm{T} 3$ control group.

The prevalence rate of successful patients in the long term (at T3) was calculated in the treatment group. As reported earlier, an unsuccessful outcome of treatment was defined as a concurrent Class 111 permanent molar relationship and a negative overjet. ${ }^{21}$ The prevalence 
Table I. Demographics for the treated and the control groups

\begin{tabular}{|c|c|c|c|c|c|c|c|c|c|c|c|}
\hline \multicolumn{4}{|c|}{ Treated group (9 male, 13 female) } & \multicolumn{4}{|c|}{ Control group T1-T2 (9 male, 7 female) } & \multicolumn{4}{|c|}{ Control group T1-T3 (8 male, 5 female) } \\
\hline $\begin{array}{l}\text { Period/ } \\
\text { interval }\end{array}$ & $n$ & Mean (y) & $S D(y)$ & $\begin{array}{l}\text { Period/ } \\
\text { interval }\end{array}$ & $n$ & Mean $(y)$ & $S D(y)$ & $\begin{array}{l}\text { Period/ } \\
\text { interval }\end{array}$ & $n$ & Mean $(y)$ & $S D(y)$ \\
\hline $\mathrm{T} 1$ & 22 & 9.2 & 1.6 & T1 & 16 & 8.6 & 1.8 & $\mathrm{~T} 1$ & 13 & 8.4 & 0.9 \\
\hline T2 & 22 & 14.5 & 1.9 & T2 & 16 & 14.8 & 2.1 & & & & \\
\hline T3 & 22 & 18.7 & 2.1 & & & & & T3 & 13 & 17.5 & 1.1 \\
\hline T1-T2 & 22 & 5.3 & 1.9 & $\mathrm{~T} 1-\mathrm{T} 2$ & 16 & 6.2 & 2.0 & & & & \\
\hline T1-T3 & 22 & 9.4 & 2.5 & & & & & T1-T3 & 13 & 9.5 & 1.8 \\
\hline
\end{tabular}

There were no significant differences either between the treated group and the respective control group at any age period or observation interval (Mann-Whitney $\mathrm{U}$ test, $P<0.05$ ), or sex distribution ( $\mathrm{z}$ test on proportions, $P<0.05$ ).

rate of compliance was calculated in treatment group, and the association with the long-term outcome of treatment was appraised with the chi-square test.

The power of the study was calculated on the basis of the difference between the treated and control groups for a relevant cephalometric variable (ANB angle) as reported in a previous longitudinal investigation of similar nature and on the basis of the standard deviation of this difference. ${ }^{4}$ The power exceeded 0.90 at an $\alpha$ level of 0.05 .

The method error was determined with 15 lateral cephalograms, selected randomly, traced and measured twice within a week by the same operator (C.M.). The measurements at both times for each patient were analyzed with the intraclass correlation coefficient, which varied between 0.966 for the SNB angle and 0.995 for the inclination of the maxillary incisor to the SN line. These values indicated a high level of intraobserver agreement. Linear measurement errors averaged 0.3 $\mathrm{mm}$ (SD, $0.8 \mathrm{~mm}$ ), and angular measurement errors averaged $0.4^{\circ}\left(\mathrm{SD}, 0.6^{\circ}\right)$.

The stage of cervical vertebral maturation was assessed for each film in a patient's series by using the method described by Baccetti et al. ${ }^{14}$ Staging of cervical vertebrae for each cephalogram was performed by the senior author (T.B.) and then verified by 2 independent investigators (C.M., L.F.) experienced in this method.

\section{RESULTS}

The demographic data of the treated group and the control groups (T1-T2 and T1-T3) are reported in the Table 1, along with the statistical comparisons showing no significant difference for age at observation periods, age intervals, or sex distribution. The comparisons of the starting forms of the treated group vs the control group T1-T2 and the control group T1-T3 (Table 11) showed no significant differences with the exception of a smaller inclination of the maxillary incisors to $\mathrm{SN}$ in the control group T1-T2 with respect to the control group T1-T3.

The success rate in the long term (at T3) in the treated group was 16 of 22 patients, or $72.7 \%$.
Table 111 reports the treatment effects when comparing the T1-T2 changes in the treated group vs the control group T1-T2. These effects comprise the outcomes of active RME/FM therapy followed by fixed appliances in a 2-phase treatment protocol. The treated group showed a significantly greater amount of maxillary advancement (Point $A$ to nasion perp, $1.4 \mathrm{~mm}$ ), along with significant decrements in the size and sagittal position of the mandible (Co-Gn, $-4.2 \mathrm{~mm} ; \mathrm{SNB},-1.6^{\circ} ; \mathrm{Pg}$ to nasion perp, $-2.8 \mathrm{~mm})$. The sagittal maxillomandibular skeletal variables all showed highly significant improvements in the treated group vs the control group (Wits appraisal, $3.9 \mathrm{~mm}$; max/mand differential, -5.2 mm; ANB angle, $2.1^{\circ}$ ).

Overjet and molar relationship improved significantly in the treated group vs the control groups (2.5 and -4.9 $\mathrm{mm}$, respectively), with a significantly reduced amount of maxillary incisor proclination in the treated group (U1 to SN, $-6.5^{\circ}$ ).

Table IV reports the overall long-term treatment and posttreatment effects when comparing the T1-T3 changes in the treated group vs the control group T1T3. These effects comprised the outcomes of the 2phase treatment protocol followed by an average of 5 years of posttreatment observation. The T3 observation was taken approximately 8 years after the completion of the orthopedic portion of therapy (RME/FM).

In the long term, no significant differences in maxillary changes were recorded, whereas the treated group still showed a significant decrement in the sagittal position of the mandible (SNB, $-2.0^{\circ}$ ). The sagittal maxillomandibular skeletal variables all maintained significant improvements in the treated group vs the control group (Wits, $3.0 \mathrm{~mm}$; max/mand differential, -3.7 mm; ANB angle, $1.4^{\circ}$ ). The molar relationship improved significantly in the treated group (3.2 $\mathrm{mm}$ more than the T1T3 control group).

The analysis of compliance of the treated subjects during the orthopedic therapy (use of the facial mask) showed that none had a "poor" degree of cooperation; 
Table II. Analysis of starting forms

\begin{tabular}{|c|c|c|c|c|c|c|c|c|c|}
\hline \multirow[b]{2}{*}{ Cephalometric measures } & \multicolumn{2}{|c|}{$\begin{array}{c}\text { Treated } \\
\text { group (TG) } \\
n=22\end{array}$} & \multicolumn{2}{|c|}{$\begin{array}{c}\text { Control group } \\
\text { (CG T1-T2) } \\
n=16\end{array}$} & \multicolumn{2}{|c|}{$\begin{array}{c}\text { Control group } \\
\text { (CG T1-T3) } \\
n=13\end{array}$} & \multirow{2}{*}{$\begin{array}{c}T G \text { vs } C G \\
T 1-T 2\end{array}$} & \multirow{2}{*}{$\begin{array}{c}T G \text { vs } C G \\
T 1-T 3\end{array}$} & \multirow{2}{*}{$\begin{array}{c}\text { CG T1-T2 vs } \\
\text { CG T1-T3 }\end{array}$} \\
\hline & Mean & $S D$ & Mean & $S D$ & Mean & $S D$ & & & \\
\hline \multicolumn{10}{|l|}{ Cranial base } \\
\hline Cranial flexure $\left(^{\circ}\right)$ & 130.5 & 5.8 & 127.2 & 4.9 & 126.3 & 3.6 & NS & NS & NS \\
\hline \multicolumn{10}{|l|}{ Maxillary skeletal } \\
\hline Co-point A (mm) & 81.6 & 4.9 & 80.2 & 5.4 & 79.5 & 4.8 & NS & NS & NS \\
\hline SNA $\left({ }^{\circ}\right)$ & 79.8 & 4.3 & 79.7 & 3.5 & 80.1 & 2.8 & NS & NS & NS \\
\hline Point A to nasion perp (mm) & 0.0 & 3.0 & -1.4 & 2.1 & -1.5 & 1.9 & NS & NS & NS \\
\hline \multicolumn{10}{|l|}{ Mandibular skeletal } \\
\hline Co-Gn (mm) & 109.3 & 7.6 & 108.9 & 8.7 & 106.5 & 7.7 & NS & NS & NS \\
\hline SNB $\left(^{\circ}\right)$ & 79.5 & 3.7 & 80.2 & 3.9 & 79.7 & 3.5 & NS & NS & NS \\
\hline Pg to nasion perp (mm) & -1.0 & 5.2 & -1.6 & 7.1 & -2.9 & 7.2 & NS & NS & NS \\
\hline Gonial angle $\left({ }^{\circ}\right)$ & 127.1 & 4.3 & 131.2 & 6.0 & 129.3 & 6.3 & NS & NS & NS \\
\hline \multicolumn{10}{|l|}{ Maxillary/mandibular } \\
\hline Wits appraisal (mm) & -6.1 & 2.2 & -7.0 & 3.1 & -6.6 & 3.8 & NS & NS & NS \\
\hline Max/mand differential (mm) & 27.8 & 4.7 & 28.6 & 6.0 & 27.0 & 6.2 & NS & NS & NS \\
\hline ANB $\left(^{\circ}\right)$ & 0.4 & 1.8 & -0.5 & 2.7 & 0.4 & 2.6 & NS & NS & NS \\
\hline \multicolumn{10}{|l|}{ Vertical skeletal } \\
\hline FH to palatal plane $\left(^{\circ}\right)$ & -1.7 & 2.2 & -2.0 & 3.4 & -0.7 & 4.0 & NS & NS & NS \\
\hline MPA $\left({ }^{\circ}\right)$ & 25.5 & 4.5 & 28.7 & 5.6 & 27.9 & 6.1 & NS & NS & NS \\
\hline ANS to Me (mm) & 63.2 & 5.4 & 61.1 & 4.2 & 60.9 & 4.4 & NS & NS & NS \\
\hline \multicolumn{10}{|l|}{ Interdental } \\
\hline Overjet (mm) & 0.1 & 1.6 & -0.8 & 1.7 & -0.6 & 1.8 & NS & NS & NS \\
\hline Overbite (mm) & 0.3 & 1.6 & 0.5 & 1.8 & -0.4 & 1.8 & NS & NS & NS \\
\hline Molar relationship (mm) & 4.1 & 1.7 & 5.4 & 1.6 & 5.2 & 2.0 & NS & NS & NS \\
\hline \multicolumn{10}{|l|}{ Dentoalveolar } \\
\hline U1 to $\mathrm{SN}\left({ }^{\circ}\right)$ & 107.0 & 10.6 & 99.9 & 8.7 & 118.7 & 19.7 & NS & NS & $*$ \\
\hline $\mathrm{L} 1$ to $\mathrm{MPA}\left({ }^{\circ}\right)$ & 84.2 & 5.3 & 82.5 & 7.6 & 85.2 & 8.0 & NS & NS & NS \\
\hline
\end{tabular}

7 had "moderate" compliance, and the remaining 15 patients had "good" compliance. As a result, therefore, cooperation was good in $68.2 \%$ of the patients.

When compliance was evaluated in association with the long-term outcomes of therapy (at T3), 5 patients with moderate compliance had long-term unfavorable results, whereas only 2 patients with moderate compliance had a successful outcome of treatment at T3. The association between the degree of compliance and the long-term clinical success of therapy was highly significant (chi-square, 7.061; $P=0.008$ ).

\section{DISCUSSION}

We analyzed for the first time the long-term effects of RME/FM therapy in growing subjects with Class 111 malocclusion. The main features of our longitudinal study were the following.

1. Patients were treated with RME/FM consecutively and enrolled in the study regardless of successful outcomes. A posttreatment observation (T2) was taken approximately 4 years after the end of RME/ FM therapy, and the long-term appraisal (T3) was approximately 8 years after the end of the orthopedic treatment.

2. The Class 111 patients were treated with the orthopedic protocol at a prepubertal stage of skeletal maturity (CS 1 or CS 2). All patients were postpubertal at T2 (CS 4-CS 6), and they had completed the active circumpubertal growth at the T3 observation (at least 2 years in CS 6).

3. The control samples consisted of subjects with untreated Class 111 malocclusion followed longitudinally, and they matched the treated group as to dentoskeletal Class 111 disharmony, age intervals, skeletal maturations at different time points, and sex distribution (Tables 1 and 11 ).

The results of the T1-T2 longitudinal observation (Table III) can be regarded as the short-term outcomes 
Table III. Analysis of treatment effects (T1-T2) and comparisons (Mann-Whitney U test, $P<0.05$ ) of T1-T2 changes in the treated group vs T1-T2 changes in the control group T1-T2

\begin{tabular}{|c|c|c|c|c|c|c|c|}
\hline \multirow[b]{2}{*}{ Cephalometric measurement } & \multicolumn{2}{|c|}{ Treated group (TG) $n=22$} & \multicolumn{2}{|c|}{ Control group (CG T1-T2) $n=16$} & \multicolumn{3}{|c|}{$T G v s$ CG T1-T2 } \\
\hline & Mean & $S D$ & Mean & $S D$ & Net difference & $\mathrm{P} v c$ & \\
\hline \multicolumn{8}{|l|}{ Cranial base } \\
\hline Cranial flexure $\left(^{\circ}\right)$ & -0.2 & 2.8 & -0.2 & 2.0 & 0.0 & 0.919 & NS \\
\hline \multicolumn{8}{|l|}{ Maxillary skeletal } \\
\hline Co-point A (mm) & 8.3 & 4.1 & 7.3 & 2.6 & 1.0 & 0.438 & NS \\
\hline SNA $\left({ }^{\circ}\right)$ & 1.9 & 2.9 & 1.5 & 1.8 & 0.4 & 0.965 & NS \\
\hline Point A to nasion perp (mm) & 1.7 & 1.8 & 0.3 & 1.6 & 1.4 & 0.036 & * \\
\hline \multicolumn{8}{|l|}{ Mandibular skeletal } \\
\hline Co-Gn (mm) & 14.1 & 4.8 & 18.3 & 5.3 & -4.2 & 0.021 & * \\
\hline SNB $\left({ }^{\circ}\right)$ & 2.0 & 3.1 & 3.6 & 2.3 & -1.6 & 0.042 & * \\
\hline Pg to nasion perp (mm) & 4.2 & 4.0 & 7.0 & 4.0 & -2.8 & 0.048 & * \\
\hline Gonial angle $\left(^{\circ}\right)$ & -3.0 & 3.3 & -2.5 & 3.5 & -0.5 & 0.827 & NS \\
\hline \multicolumn{8}{|l|}{ Maxillary/mandibular } \\
\hline Wits appraisal (mm) & 1.1 & 2.6 & -2.8 & 1.8 & 3.9 & 0.000 & $\dagger$ \\
\hline Max/mand differential (mm) & 5.8 & 3.3 & 11.0 & 3.3 & -5.2 & 0.000 & $\dagger$ \\
\hline ANB $\left(^{\circ}\right)$ & 0.0 & 1.4 & -2.1 & 1.6 & 2.1 & 0.000 & $\dagger$ \\
\hline \multicolumn{8}{|l|}{ Vertical skeletal } \\
\hline FH to palatal plane $\left({ }^{\circ}\right)$ & 0.0 & 1.9 & -0.5 & 1.9 & 0.5 & 0.137 & NS \\
\hline MPA $\left({ }^{\circ}\right)$ & -2.2 & 3.0 & -2.3 & 3.4 & 0.1 & 0.849 & NS \\
\hline ANS to Me (mm) & 6.5 & 2.3 & 8.2 & 3.8 & -1.7 & 0.060 & NS \\
\hline \multicolumn{8}{|l|}{ Interdental } \\
\hline Overjet (mm) & 1.8 & 2.6 & -0.7 & 3.1 & 2.5 & 0.037 & * \\
\hline Overbite (mm) & 1.4 & 1.4 & 0.6 & 1.9 & 0.8 & 0.161 & NS \\
\hline Molar relationship (mm) & -0.5 & 1.8 & 4.4 & 2.5 & -4.9 & 0.000 & $\dagger$ \\
\hline \multicolumn{8}{|l|}{ Dentoalveolar } \\
\hline $\mathrm{U} 1$ to $\mathrm{SN}\left({ }^{\circ}\right)$ & 2.4 & 8.9 & 8.9 & 6.1 & -6.5 & 0.024 & * \\
\hline $\mathrm{L} 1$ to $\mathrm{MPA}\left({ }^{\circ}\right)$ & -0.5 & 3.9 & -1.0 & 5.7 & 0.5 & 0.589 & NS \\
\hline
\end{tabular}

of a 2-phase treatment protocol incorporating an orthopedic treatment phase (RME/FM) followed by fixed appliances in the permanent dentition. Significant improvements in all cephalometric measures for intermaxillary sagittal skeletal relationships were recorded in the treatment group during the T1-T2 interval. When they were compared with the untreated controls, the Wits appraisal improved by $3.9 \mathrm{~mm}$, the maxillomandibular differential improved by $5.2 \mathrm{~mm}$, and the ANB angle improved by $2.1^{\circ}$. Both maxillary and mandibular changes contributed to the favorable intermaxillary outcomes in the short term (Point A to nasion perp improved by $1.4 \mathrm{~mm}$, and Co-Gn decreased by $4.2 \mathrm{~mm}$, SNB angle decreased by $1.6^{\circ}$, and $\mathrm{Pg}$ to nasion perp decreased by $2.8 \mathrm{~mm}$ over the controls). At the occlusal level, overjet correction was $2.5 \mathrm{~mm}$, and the correction in molar relationships was $4.9 \mathrm{~mm}$. No significant changes were recorded in the vertical skeletal or dental relationships. These short-term postpubertal treatment outcomes were similar to those reported by Westwood et al, ${ }^{4}$ who implemented a comparable methodology of investigation.
The analysis of the T1-T3 results (Table IV) after active circumpubertal growth (average age, 18.5 years) showed that intermaxillary sagittal skeletal relationships still exhibited favorable changes compared with the untreated Class 111 controls. Substantial favorable modifications were recorded in the mandible $(-3.9 \mathrm{~mm}$ for $\mathrm{Co}-\mathrm{Gn}$ in the treated subjects vs the controls, -1.8 $\mathrm{mm}$ for Pg to nasion perp, and $-2^{\circ}$ for SNB). These mandibular changes accounted for most of the intermaxillary outcomes. No significant improvements in the maxillary measurements were found in the treated sample in the long term. More than $3 \mathrm{~mm}$ of molar correction was still assessed during the T1-T3 interval.

The long-term appraisal of the outcomes of RME/FM therapy failed to show some of the craniofacial changes that have been described in previous controlled trials reporting the short-term changes with the same treatment protocol. ${ }^{4,22}$ From T1 to T3, the treatment group did not show a significant amount of closure of the gonial angle (a growth modification that has been advocated as a favorable mechanism to limit linear increases of the mandible along $\mathrm{Co}-\mathrm{Gn}^{23}$ ) or any 
Table IV. Analysis of overall long-term effects (T1-T3) and comparisons (Mann-Whitney U test, $P<0.05$ ) of T1-T3 changes in the treated group vs T1-T3 changes in the control group T1-T3

\begin{tabular}{|c|c|c|c|c|c|c|c|}
\hline \multirow[b]{2}{*}{ Cephalometric measurement } & \multicolumn{2}{|c|}{ Treated group (TG) $n=22$} & \multicolumn{2}{|c|}{ Control group (CG T1-T3) $n=13$} & \multicolumn{3}{|c|}{$T G$ vs $C G T 1-T 3$} \\
\hline & Mean & $S D$ & Mean & $S D$ & Net difference & $\mathrm{P} v c$ & \\
\hline \multicolumn{8}{|l|}{ Cranial base } \\
\hline Cranial flexure $\left(^{\circ}\right)$ & -0.2 & 2.9 & -0.9 & 2.1 & 0.7 & 0.339 & NS \\
\hline \multicolumn{8}{|l|}{ Maxillary skeletal } \\
\hline Co-point A (mm) & 10.8 & 3.9 & 10.9 & 4.8 & -0.1 & 0.891 & NS \\
\hline SNA $\left({ }^{\circ}\right)$ & 1.9 & 2.6 & 2.1 & 2.8 & -0.2 & 0.413 & NS \\
\hline Point A to nasion perp (mm) & 1.1 & 2.6 & 0.9 & 2.8 & 0.2 & 0.891 & NS \\
\hline \multicolumn{8}{|l|}{ Mandibular skeletal } \\
\hline Co-Gn (mm) & 20.6 & 6.3 & 24.5 & 6.9 & -3.9 & 0.172 & NS \\
\hline SNB $\left({ }^{\circ}\right)$ & 2.3 & 3.0 & 4.3 & 2.7 & -2.0 & 0.048 & * \\
\hline Pg to nasion perp (mm) & 5.8 & 6.0 & 7.6 & 5.1 & -1.8 & 0.322 & NS \\
\hline Gonial angle $\left(^{\circ}\right)$ & -4.7 & 3.8 & -4.3 & 3.7 & -0.4 & 0.609 & NS \\
\hline \multicolumn{8}{|l|}{ Maxillary/mandibular } \\
\hline Wits appraisal (mm) & 0.1 & 3.7 & -2.9 & 3.7 & 3.0 & 0.017 & * \\
\hline Max/mand differential (mm) & 9.8 & 4.7 & 13.5 & 4.5 & -3.7 & 0.024 & * \\
\hline ANB $\left(^{\circ}\right)$ & -0.8 & 1.7 & -2.2 & 2.0 & 1.4 & 0.044 & * \\
\hline \multicolumn{8}{|l|}{ Vertical skeletal } \\
\hline FH to palatal plane $\left({ }^{\circ}\right)$ & 0.9 & 2.5 & 0.9 & 3.1 & 0.0 & 0.707 & NS \\
\hline MPA $\left({ }^{\circ}\right)$ & -3.2 & 3.6 & -2.4 & 4.1 & -0.8 & 0.657 & NS \\
\hline ANS to Me (mm) & 9.8 & 3.0 & 12.5 & 3.8 & -2.7 & 0.052 & NS \\
\hline \multicolumn{8}{|l|}{ Interdental } \\
\hline Overjet (mm) & 1.1 & 3.2 & -0.1 & 2.6 & 1.2 & 0.527 & NS \\
\hline Overbite (mm) & 1.2 & 1.5 & 0.7 & 2.2 & 0.5 & 0.388 & NS \\
\hline Molar relationship (mm) & 0.7 & 2.3 & 3.9 & 2.4 & -3.2 & 0.000 & $\dagger$ \\
\hline \multicolumn{8}{|l|}{ Dentoalveolar } \\
\hline $\mathrm{U} 1$ to $\mathrm{SN}\left({ }^{\circ}\right)$ & 3.1 & 9.8 & 8.8 & 8.8 & -5.7 & 0.095 & NS \\
\hline $\mathrm{L} 1$ to $\mathrm{MPA}\left({ }^{\circ}\right)$ & 0.6 & 4.4 & -1.7 & 5.0 & 2.3 & 0.227 & NS \\
\hline
\end{tabular}

significant improvement in the sagittal position of the maxilla, as described by Westwood et $\mathrm{al}^{4}$ and Pangrazio-Kulbersh et al. ${ }^{22}$ However, Franchi et al ${ }^{7}$ showed that both of these craniofacial changes are induced by early, prepubertal orthopedic treatment of Class 111 disharmony, but they are not associated with later treatment, during or after puberty. Our study included patients who underwent orthopedic treatment before puberty ( 14 patients) and at puberty ( 8 patients). Therefore, the lack of a specific prepubertal treatment in this study might explain the lack of significant results in terms of maxillary or gonial angle changes in the long term.

The favorable long-term outcomes in terms of mandibular position in the patients treated with the orthopedic protocol could be associated with changes in the glenoid fossa that have been described previously for chincap therapy ${ }^{24}$ and miniplates and Class 111 elastics protocol. ${ }^{25} \mathrm{~A}$ laminagraphic evaluation of temporomandibular joint changes after chincap therapy showed deepening and widening of the mandibular fossa, and narrowing of the clearance between the condyle and the fossa. ${ }^{24}$ No tendency to an increase in the vertical skeletal relationships was recorded in the treated group at either the T2 or T3 observations, in contrast with previous findings by Macdonald et al. ${ }^{8}$ The use of a correct downward inclination of the extraoral elastics of the facial mask might have accounted for the lack of a bite opening tendency in the treated group. ${ }^{26}$

When individual patient data were analyzed, we found that over $70 \%$ of the patients (16 of 22) could be considered clinically successful in the long term, whereas less than 30\% of them ( 6 of 22) were unsuccessful at T3 because of relapse in the occlusal relationships. The prevalence rate for the long-term success of RME/ FM therapy of Class 111 malocclusion appears as a favorable result, and it is similar to the success rates reported in other studies on orthopedic Class 111 treatment that included a posttreatment interval (76\% according to Westwood et $\mathrm{al}^{4} ; 75 \%$ according to Ngan et $\mathrm{al}^{9}$ and Wells et $\mathrm{al}^{6} ; 67 \%$ according to Hägg et $\mathrm{al}^{5}$ ).

During the posttreatment interval, the outcomes of RME/FM therapy did not show a notable trend of relapse, with the exception of the maxillary measures. It 
appears that the immediate posttreatment changes during the pubertal growth spurt (when the mandible has a longer and more intense period of growth in Class 111 vs Class 1 subjects ${ }^{11,27}$ ) have the greatest unfavorable impact on treatment outcomes in the mandibular and intermaxillary measurements. More evidence it this regard can be derived from the study by Westwood et al. ${ }^{4}$ Once the pubertal growth spurt is over, the relapse tendency after orthopedic Class 111 treatment becomes more modest.

Since the facial mask is a removable appliance, the issue of patient compliance deserves to be investigated in relation to the long-term treatment outcomes. Interestingly, 5 of the 6 patients with unsuccessful results at T3 had a modest degree of compliance during active therapy with the facial mask, whereas just 2 of the 16 successful patients had poor compliance. However, significant differences were found in the pretreatment craniofacial features of unsuccessful vs successful patients that can explain further the failure of therapy on the basis of the severity and specific characteristics of the initial dentoskeletal disharmony. The unsuccessful patients showed a significantly greater gonial angle $\left(+3.8^{\circ}\right)$, a downward inclination of the mandibular plane to Frankfort horizontal $\left(+4.1^{\circ}\right)$, and a mesial molar relationship $(+1.5 \mathrm{~mm})$. Therefore, both patient compliance and pretreatment dentoskeletal features can be regarded as significant factors influencing the long-term results of RME/FM treatment.

The outcomes of this study can be compared with those of other long-term controlled studies on orthopedic treatment of Class 111 malocclusion in the literature. The results of the RME/FM study by PangrazioKulbersh et $\mathrm{al}^{22}$ are similar to those reported here, with the exception of less posttreatment relapse tendency in the skeletal sagittal position of the maxilla. The results of the long-term study on the FR-3 appliance indicated that the treated group showed a significant increase in midfacial length compared with the controls, without any significant change in the sagittal position of the maxilla or in the mandibular dimensions. ${ }^{28}$ Sugawara et $\mathrm{al}^{12}$ found that most of the favorable changes induced by chincaps reverted completely in the long term. With respect to both the FR-3 and chincap therapies, however, the RME/FM protocol has a significantly shorter duration of active treatment. Whereas chincap wear was on average 4.5 years (range, 1-9.5 years) ${ }^{12}$ and FR-3 wear consisted of about 2.5 years of active full-time appliance wear followed by at least 3 years of part-time wear, ${ }^{28}$ the RME/FM protocol entails a much smaller "burden of treatment." The analysis of active treatment duration in this study showed that the orthopedic appliance was worn for an average of 1.1 years (range, 4 months- 2 years). Only 1 patient resumed facial mask wear during phase 2 treatment. Therefore, RME/FM treatment is an efficient orthopedic protocol even when considering the possibility of adding a second phase of treatment with comprehensive fixed appliance therapy.

\section{CONCLUSIONS}

1. In the long term, RME/FM therapy of subjects with Class III dentoskeletal disharmony led to successful outcomes in about 73\% of the patients.

2. Approximately 8 years after the end of RME/FM therapy, the patients still showed significantly improved sagittal dentoskeletal relationships. These favorable changes were mainly due to significant improvements in the sagittal position of the mandible, but the maxillary changes reverted completely in the long term.

3. RME/FM therapy of Class 111 malocclusion did not induce a tendency of bite opening or increased vertical relationship.

4. The long-term results of RME/FM therapy are influenced by the patient's compliance and pretreatment dentoskeletal features (Class 111 disharmony associated with increased facial divergence).

\section{REFERENCES}

1. McNamara JA Jr, Brudon WL. Orthodontics and dentofacial orthopedics. Ann Arbor, Mich: Needham Press; 2001. p. 375-85.

2. Keim RG, Gottlieb EL, Nelson AH, Vogels DS 3rd. 2008 JCO study of orthodontic diagnosis and treatment procedures, part 1: results and trends. J Clin Orthod 2008;42:625-40.

3. Toffol LD, Pavoni C, Baccetti T, Franchi L, Cozza P. Orthopedic treatment outcomes in Class 111 malocclusion. A systematic review. Angle Orthod 2008;78:561-73.

4. Westwood PV, McNamara JA Jr, Baccetti T, Franchi L, Sarver DM. Long-term effects of Class 111 treatment with rapid maxillary expansion and facemask therapy followed by fixed appliances. Am J Orthod Dentofacial Orthop 2003;123:306-20.

5. Hägg U, Tse A, Bendeus M, Rabie AB. Long-term follow-up of early treatment with reverse headgear. Eur J Orthod 2003;25:95-102.

6. Wells AP, Sarver DM, Proffit WR. Long-term efficacy of reverse pull headgear therapy. Angle Orthod 2006;76:915-22.

7. Franchi L, Baccetti T, McNamara JA. Postpubertal assessment of treatment timing for maxillary expansion and protraction therapy followed by fixed appliances. Am J Orthod Dentofacial Orthop 2004;126:555-68.

8. Macdonald KE, Kapust AJ, Turley PK. Cephalometric changes after the correction of Class 111 malocclusion with maxillary expansion/ facemask therapy. Am J Orthod Dentofacial Orthop 1999;116: 13-24.

9. Ngan PW, Hägg U, Yiu C, Wei SH. Treatment response and long-term dentofacial adaptations to maxillary expansion and protraction. Semin Orthod 1997;3:255-64.

10. Gallagher RW, Miranda F, Buschang PH. Maxillary protraction: treatment and posttreatment effects. Am J Orthod Dentofacial Orthop 1998;113:612-9. 
11. Kuc-Michalska M, Baccetti T. Duration of the pubertal peak in skeletal Class 1 and Class 111 subjects. Angle Orthod 2010;80:54-7.

12. Sugawara J, Asano T, Endo N, Mitani H. Long-term effects of chincap therapy on skeletal profile in mandibular prognathism. Am J Orthod Dentofacial Orthop 1990;98:127-33.

13. Baccetti T, Rey D, Oberti G, Stahl F, McNamara JA Jr. Long-term outcomes of Class 111 treatment with mandibular cervical headgear followed by fixed appliances. Angle Orthod 2009;79:828-34.

14. Baccetti T, Franchi L, McNamara JA Jr. The cervical vertebral maturation (CVM) method for the assessment of optimal treatment timing in dentofacial orthopedics. Semin Orthod 2005;11:119-29.

15. McNamara JA Jr. An orthopedic approach to the treatment of Class 111 malocclusion in young patients. J Clin Orthod 1987;21:598-608.

16. Slakter MJ, Albino JE, Fox RN, Lewis EA. Reliability and stability of the orthodontic patient cooperation scale. Am J Orthod 1980;78: 559-63.

17. Tollaro 1, Baccetti T, Franchi L. Mandibular skeletal changes induced by early functional treatment of Class 111 malocclusion: a superimposition study. Am J Orthod Dentofacial Orthop 1995;108: 525-32.

18. Jacobson A. The "Wits" appraisal of jaw disharmony. Am J Orthod 1975;67:125-38.

19. McNamara JA Jr. A method of cephalometric evaluation. Am J Orthod 1984;86:449-69.

20. Steiner CC. Cephalometrics for you and me. Am J Orthod 1953;39: 729-55.
21. Baccetti T, Franchi L, McNamara JA Jr. Cephalometric variables predicting the long-term success or failure of combined rapid maxillary expansion and facial mask therapy. Am J Orthod Dentofacial Orthop 2004;126:16-22.

22. Pangrazio-Kulbersh V, Berger JL, Janisse FN, Bayirli B. Long-term stability of Class 111 treatment: rapid palatal expansion and protraction facemask vs LeFort 1 maxillary advancement osteotomy. Am J Orthod Dentofacial Orthop 2007;131:7.e9-719.

23. Lavergne J, Gasson N. Operational definitions of mandibular morphogenetic and positional rotations. Scand J Dent Res 1977;85: 185-92.

24. Mimura H, Deguchi T. Morphologic adaptation of temporomandibular joint after chincup therapy. Am J Orthod Dentofacial Orthop 1996;110:541-6.

25. Heymann GC, Cevidanes L, Cornelis M, De Clerck HJ, Tulloch JF. Three-dimensional analysis of maxillary protraction with intermaxillary elastics to miniplates. Am J Orthod Dentofacial Orthop 2010;137:274-84.

26. Tanne K, Hiraga J, Sakuda M. Effects of directions of maxillary protraction forces on biomechanical changes in craniofacial complex. Eur J Orthod 1989;11:382-91.

27. Baccetti T, Franchi L, McNamara JA. Growth in the untreated Class 111 subject. Semin Orthod 2007;13:130-42.

28. Levin AS, McNamara JA Jr, Franchi L, Baccetti T, Fränkel C. Shortterm and long-term treatment outcomes with the FR-3 appliance of Fränkel. Am J Orthod Dentofacial Orthop 2008;134:513-24. 